\title{
THE THERMAL HISTORY OF THE UNIVERSE AND THE SPECTRUM OF RELIC RADIATION
}

\author{
R. A. SUNYAEV*
}

Institute of Applied Mathematics, USSR Academy of Sciences, Moscow, U.S.S.R.

Many of the cosmological models currently under discussion and theories of the origin of galaxies which involve antimatter, strong turbulence and so on result in significant energy release during the evolution of the Universe. It is evident that significant energy production should lead to distortions of the spectrum of the relic radiation. The absence of noticeable deviations from the Planckian spectrum enables us to set limits to the energy release in the early Universe $\left(10^{2}<z<10^{8}\right)$. But in order to have a clear picture of possible distortions, let us first review the idealized situation.

Why do we predict an exact Planckian spectrum in the idealized case of a strictly Friedmannian hot universe? The answer is not so simple! At high temperatures when many electrons and positrons are present and the density is high enough, there is plenty of bremsstrahlung radiation and absorption and obviously thermal equilibrium is established. But these relaxation processes are absent at redshifts $z<10^{8}$ due to the small concentration of protons and electrons left at this time. For the most important part of the spectrum $(h v \sim 3 k T)$ the free-free optical depth is only $10^{-2}$ integrating from $z=10^{8}$ up to the present time. But the important point is that the overall expansion does not change the Planckian character of the spectrum - only the temperature decreases like $(1+z)$. The radiation in bulk is like a 100 year old virgin - being virgin just because nobody wished to violate her ** $^{* *}$

Let us introduce parameters:

$$
\begin{aligned}
& x=\frac{h v}{k T_{\mathrm{r}}} ; \quad \Omega=\frac{\varrho}{\varrho_{\text {crit }}} \\
& \hbar=\frac{H_{0}}{50 \mathrm{~km} \mathrm{~s}^{-1} \mathrm{Mpc}^{-1}} \\
& y=\int \frac{k T_{\mathrm{r}}}{m c^{2}} \mathrm{~d} \tau_{T}=7 \times 10^{-10} \Omega \hbar^{2} z^{2}
\end{aligned}
$$

The thermal history of the Universe may be divided into 5 stages, each of which influences the final spectrum of relic radiation.

1. $z>10^{8}$

The concentrations of electrons and protons is great, and so both Compton and freefree processes lead to a Planckian spectrum independent of the magnitude of the energy release.

* Delivered by Ya. B. Zel'dovich.

** This comparison was made by Ya. B. Zel'dovich, not by the author. 
2. $3 \times 10^{5}(\Omega \hbar)^{-6 / 5}<z<10^{8}$

A significant energy release leads to the formation of a Bose-Einstein spectrum. It is important that even if the energy release is small $\left(\sim 1 \% \varepsilon_{r}\right)$, the deviation of the spectrum from the Planckian curve is large at wavelength $\lambda \approx 3.5\left(\Omega \hbar^{2}\right)^{-7 / 8} \mathrm{~cm}$. However, we can only obtain information about sufficiently large energy releases that $\left.\Delta \varepsilon / \varepsilon_{r}>4 \times 10^{-3} \Omega \hbar^{2}\right)^{7 / 8}$. There is time for smaller distortions of the spectrum to be damped out.

3. $4 \times 10^{4}\left(\Omega \hbar^{2}\right)^{-1 / 2}<z<3 \times 10^{5}\left(\Omega \hbar^{2}\right)^{-6 / 5}$

The Bose-Einstein spectrum is also formed in this period, but the distortions do not disappear no matter how small they might be.

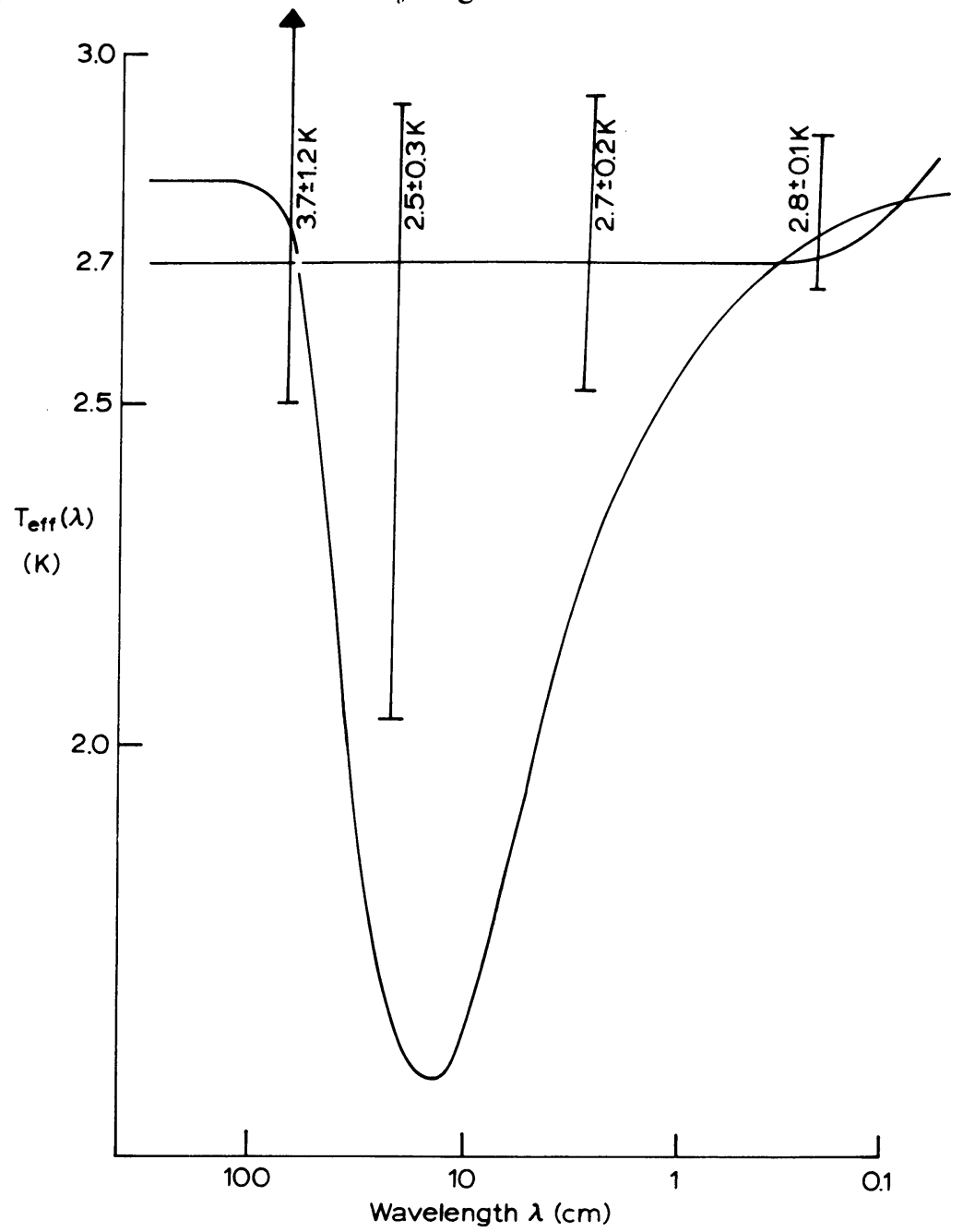

Fig. 1. The influence of the energy release $\Delta \varepsilon / \varepsilon=3.5 \%$ at $2 \times 10^{5}<z<3 \times 10^{7}$ on the relic radiation spectrum, when $\Omega=0.1$. Results of observations are given for comparison. 
4. $1400<z<4 \times 10^{4}\left(\Omega \hbar^{2}\right)^{-1 / 2}$

In this period $y<1$ and there is not enough time for the formation of a Bose-Einstein spectrum as a result of the Compton effect. The energy release, no matter what its nature is, leads to a decrease in intensity in the low-frequency region of the spectrum $\varepsilon_{v} \sim e^{-2 y} \varepsilon_{v_{0}}$ and to an increase in intensity at high frequencies (due to the Compton effect).

5. $100<z<1400$

At $z \approx 1400$ recombination of the hydrogen in the Universe takes place and the degree of ionisation of the primordial matter rapidly decreases. A large energy release will

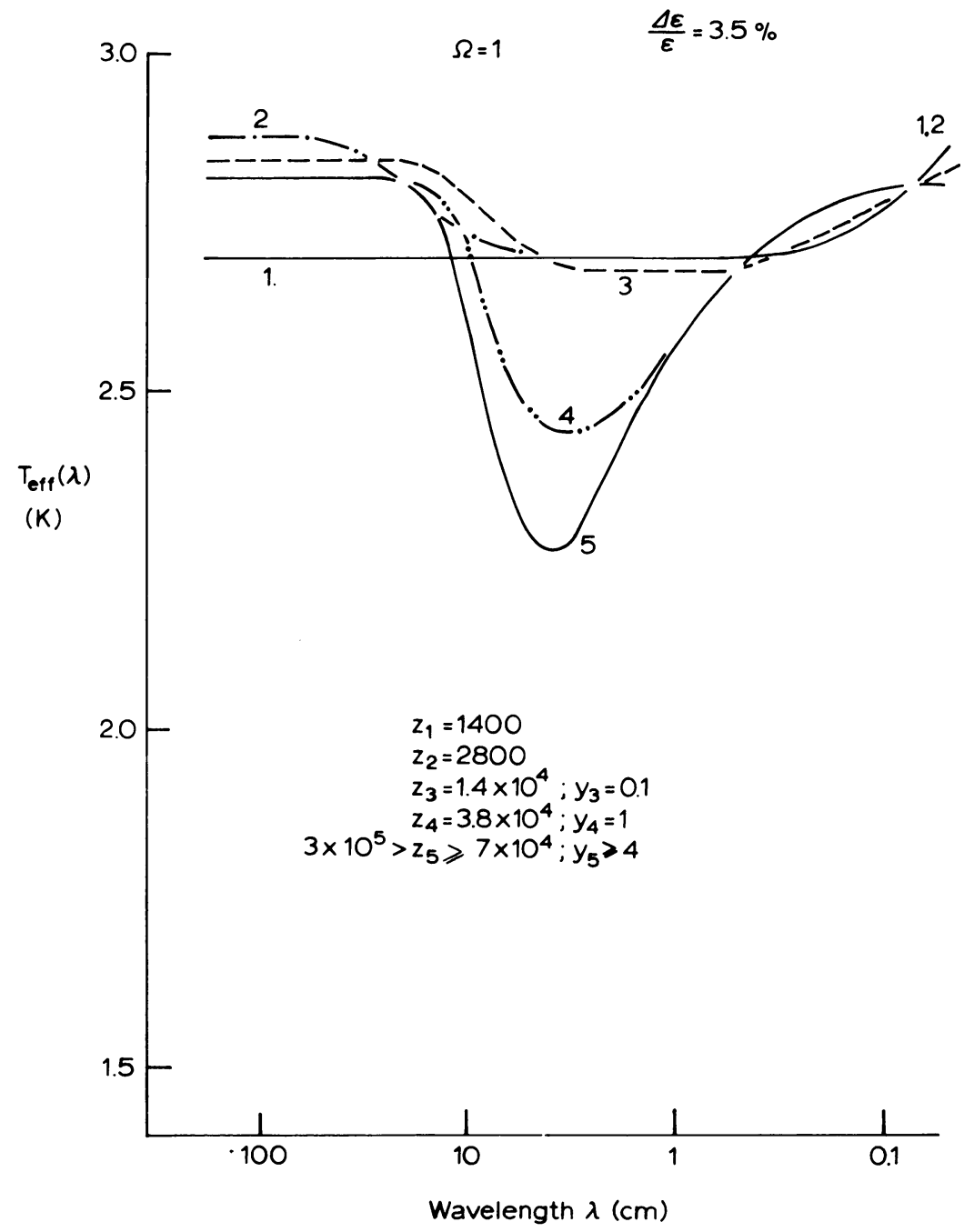

Fig. 2. The influence of the energy release $\Delta \varepsilon / \varepsilon=3.5 \%$, which heats directly the radiation at different stages in the expansion of the Universe, on the relic radiation spectrum, when $\Omega=1$. 
result in the ionisation of the plasma, i.e., it must raise the temperature above $10^{4} \mathrm{~K}$. The bremsstrahlung radiation of this hot gas may be observable at low radio frequencies.

The types of distortion expected if a moderate energy release takes place at different redshifts is illustrated in Figures 2 and 3.

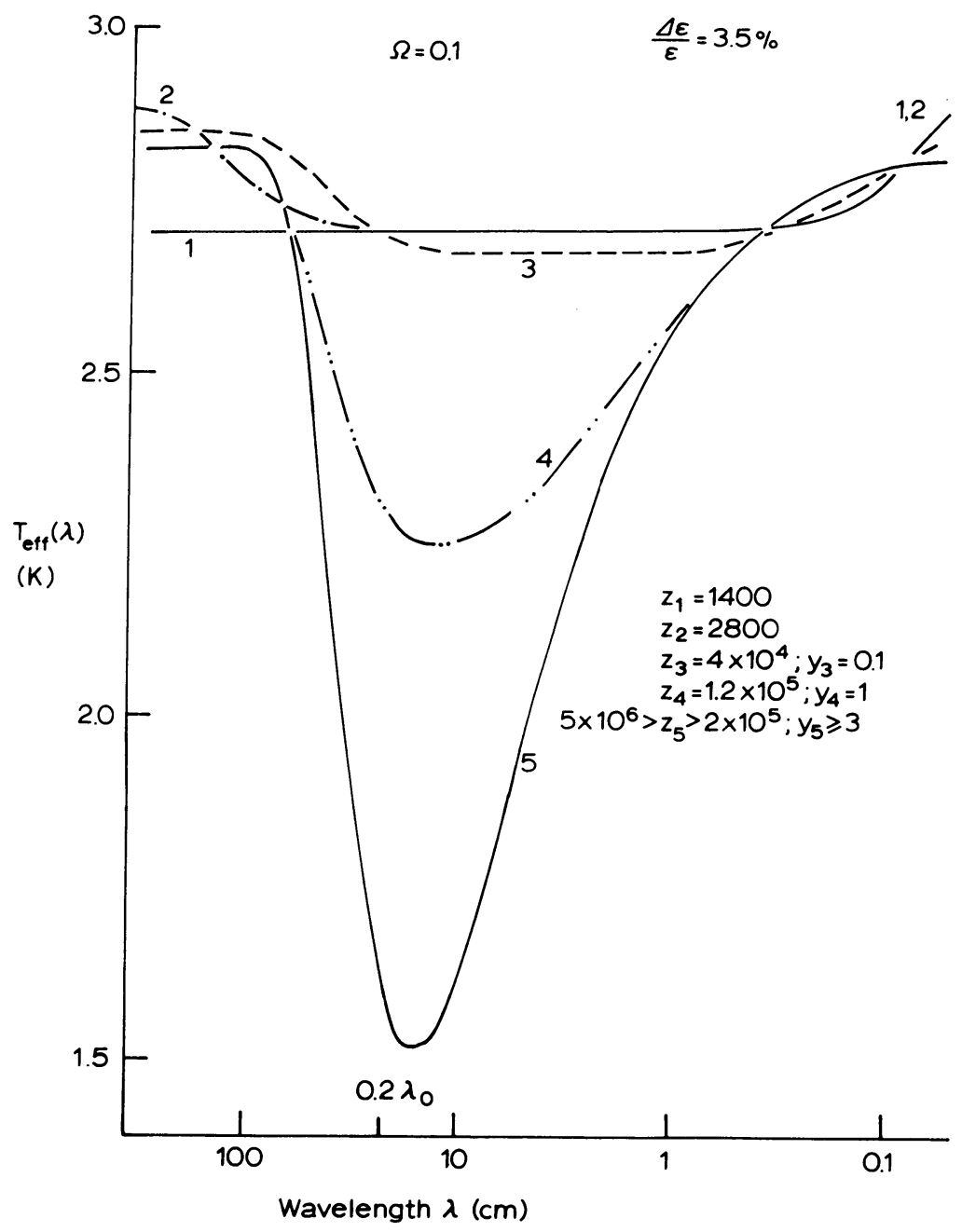

Fig. 3. The influence of the energy release $\Delta \varepsilon / \varepsilon=3.5 \%$, which heats directly the radiation at different stages in the expansion of the Universe, on the relic radiation spectrum, when $\Omega=0.1$.

The available experimental data strongly suggest the absence of deviations from a Planckian spectrum with temperature $T_{\mathrm{r}}=2.7 \mathrm{~K}$ : the experimental errors indicate that the deviations cannot be larger than $30 \%$ in the region $\lambda=50-20 \mathrm{~cm}$ or larger than $10 \%$ in the region $\lambda=20-1 \mathrm{~cm}$ (Figure 1). Measurements in the high-frequency 


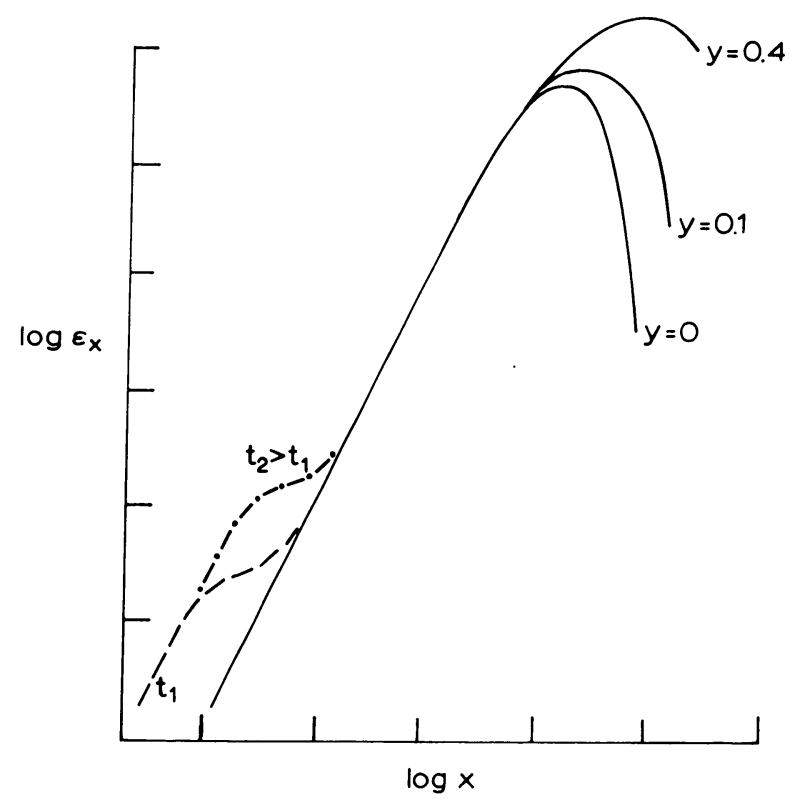

Fig. 4. Distortions of the relic radiation spectrum due to the energy release after the epoch of recombination and the jump in the spectrum due to the bremsstrahlung of plasma with temperature $T_{e}>T_{R-J}$.

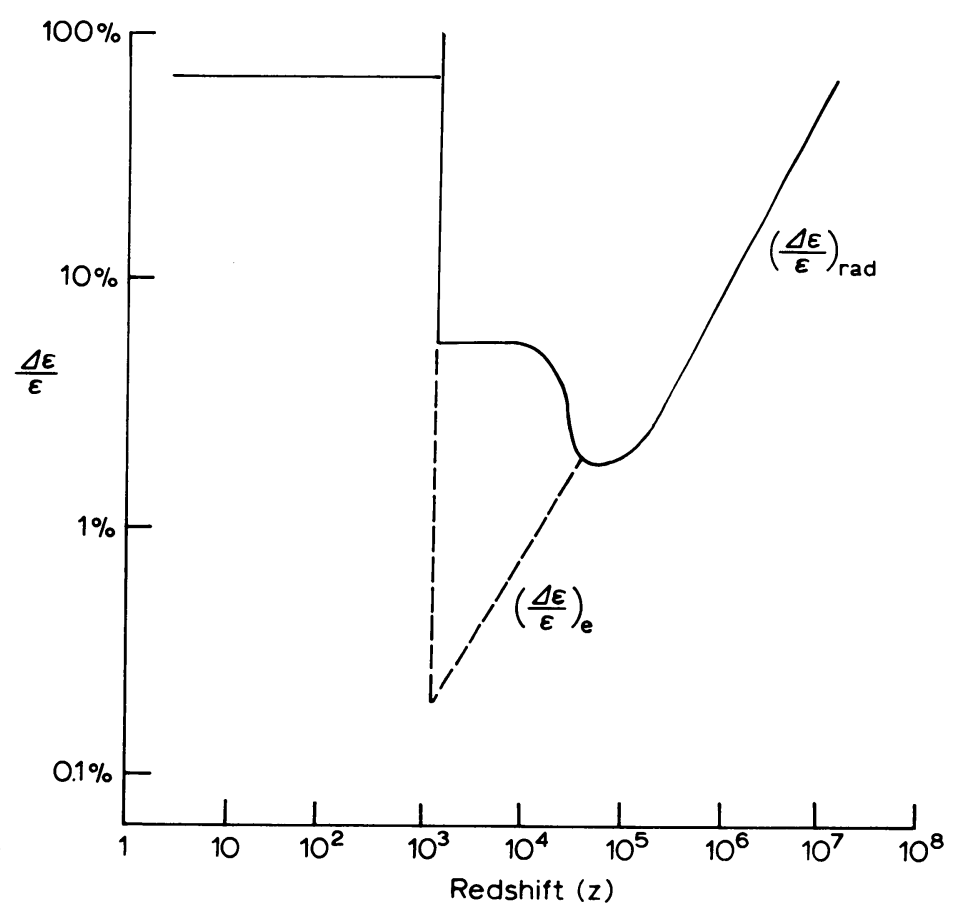

Fig. 5. Restrictions on possible values of the energy release in the Universe, which directly heats the radiation (heavy line) and the electrons (dashed line), when $\Omega=1$. 
region and data obtained from observation of interstellar molecules set a limit to the parameter $y<0.15$ and to the magnitude of the energy release $\Delta \varepsilon / \varepsilon<0.8$ during the stage $100<z<1400$ (see Figure 4).

At earlier stages of the expansion of the Universe the observations give limits $\Delta \varepsilon / \varepsilon_{r}<5 \%$ in the range $1400<z<4 \times 10^{4}$ for $\Omega \hbar^{2} \sim 1$. At $4 \times 10^{4}<z<5 \times 10^{5}$ the limitations are still more stringent: $\Delta \varepsilon / \varepsilon_{r}<1 \%$. The limits to the energy release over the whole range of redshifts, $z<10^{8}$, deduced from the above arguments are summarised in Figure 5. Of course, such limits contradict many theories in which it is supposed that in the Universe there were present large amounts of antimatter which annihilated.

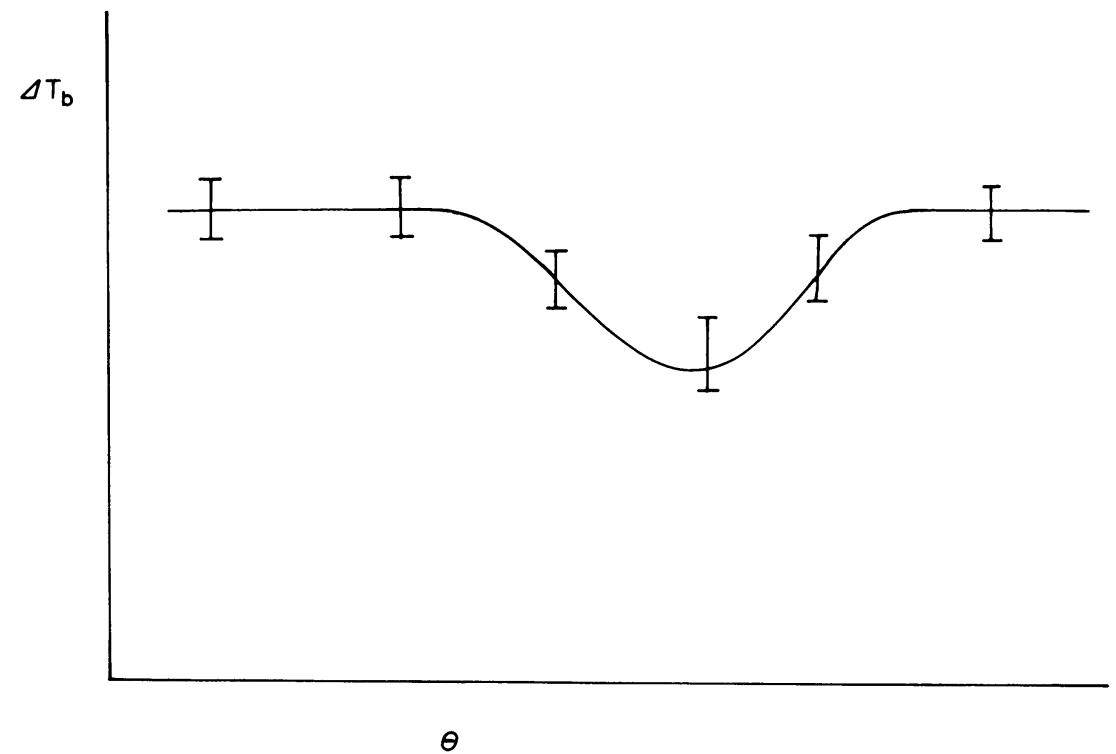

Fig. 6. The dip in brightness temperature of the relic radiation in the direction of a cluster of galaxies due to Compton scattering by hot electrons in the intergalactic gas in the cluster

(schematic diagram after Parijskij).

X-ray observations from the UHURU satellite show that many rich clusters of galaxies are powerful sources of X-ray radiation. If this X-ray radiation is the bremsstrahlung of hot intergalactic gas, then the temperature of this gas must be $T_{e} \sim 10^{8} \mathrm{~K}$ and its density $n_{e} \sim 10^{-4}-10^{-3} \mathrm{~cm}^{-3}$ (if the cluster dimensions are $\sim 10^{25}$ $\mathrm{cm})$. It can be easily seen that in this case $y \sim 10^{-4}$ to $10^{-3}$ and the temperature of the relic radiation in the direction of such clusters must decrease, the magnitude of the fluctuation being $\Delta T / T=-2 y \sim 10^{-4}-10^{-3}$. Such a decrease in the brightness temperature of the relic radiation in the direction of a given cluster of galaxies - if it exists - is difficult to explain by any other means (Figure 6).

\section{References}

I. Basic Theory of Compton Interaction

Kompaneets, A. S.: 1956, JETP 31, 876.

Weymann, R.: 1968, Astrophys. J. 145, 560. 
II. Application of the Theory to Cosmological Situations Including the Effects of Free-Free Emission and Electron Temperature Readjustment

Illarionov, A. F. and Sunyaev, R. A.: 1972, Astron. Zh. 49, 58.

Sunyaev, R. A. and Zel'dovich, Ya. B.: 1970, Astrophys. Space Sci. 7, 20.

Sunyaev, R. A. and Zel'dovich, Ya. B. : 1972, Comments Astrophys. Space Phys. 10, 21.

Zel'dovich, Ya. B. and Sunyaev, R. A.: 1969, Astrophys. Space Sci. 4, 301.

III. Coma Cluster Observations

Parijskij, Yu. N.: 1973, Astron. Zh. 50, 453.

\section{DISCUSSION}

Silk: The mass of X-ray emitting gas at $10^{8} \mathrm{~K}$ in the Coma cluster is found to be $\sim 10^{14}\left(H_{0} / 50\right)^{-5 / 2} M_{\odot}$ or about $10 \%$ of the binding mass if the gas is assumed to possess a similar distribution to that of the galaxies.

Steigman: Since $\varepsilon_{r}=$ (constant) $T_{1}^{4} e^{12 y}$ where $T_{1}=T_{0} e^{-2 y}\left(T_{1}\right.$ is the observed 'Rayleigh-Jeans' temperature), can't we set better limits to $y$ by setting limits to $\varepsilon_{r}$, say, by the limits set from Compton scattering?

Zel'dovich: Of course, if we agree with Blair that $\varepsilon_{r}$ is less than 1.2 (const) $T_{1}^{4}$ then $y \leqslant 0.08$. This limit refers to the period after reheating (or of second ionisation) of the intergalactic gas at $z_{2}<1400$. For $H_{0}=$ $=50 \mathrm{~km} \mathrm{~s}^{-1} \mathrm{Mpc}^{-1}, \tau=0.02 \Omega^{1 / 2} z_{2}^{3 / 2}$,

$$
y=\frac{k T_{e}}{n_{e} c^{2}} \tau=3 \times 10^{-12} z_{2}^{3 / 2} \Omega^{1 / 2} T_{e} .
$$

For example, if we take $z_{2}=500$ and $\Omega=0.2$ we find $T_{e}<5 \times 10^{6} \mathrm{~K}$ if $T_{e}$ is a constant or is the average value of the temperature after second ionisation. The limit is not very stringent. One should improve greatly the precision with which $y$ is known but it is likely to be limited by the existence of infrared sources.

These types of measurement exclude the possibility of strong turbulence surviving up to the epoch of recombination (for an exact treatment see Illarionov and Sunyaev: Astron. Zh., in press, preprint, 1973. The differential approach (of the type used for the Coma cluster) seems to be more sensitive for the case of late ionisation which should not be uniform in space or time of reheating.

Scheuer: What is the angular width of the depression in the microwave background which Parijskij finds in the Coma cluster?

Zel'dovich: It is definitely greater than 30 min (see references to Parijskij's work). 